\title{
An anaesthetic and intensive care perspective on infection control measures for the prevention of airborne transmission of SARS-CoV-2
}

\author{
O'Mahony, HR
}

http://hdl.handle.net/10026.1/16760

10.12968/hmed.2020.0538

British Journal of Hospital Medicine

Mark Allen Group

All content in PEARL is protected by copyright law. Author manuscripts are made available in accordance with publisher policies. Please cite only the published version using the details provided on the item record or document. In the absence of an open licence (e.g. Creative Commons), permissions for further reuse of content should be sought from the publisher or author. 
Title:

An anaesthetic and intensive care perspective on infection control measures for the prevention of airborne transmission of SARS CoV-2

Authors:

Hazel R O'Mahony ${ }^{1}$ and Daniel S Martin ${ }^{1,2}$

\section{Affiliations:}

1. Intensive Care Unit, Royal Free Hospital, Pond Street, London, NW3 2QG, UK.

2. Peninsula Medical School, University of Plymouth, John Bull Building, Tamar Science Park, Derriford, Plymouth, PL6 8BU, UK.

Corresponding Author: Hazel O'Mahony, h.o'mahony@nhs.net 07749731929 


\section{Abstract}

Guidance regarding appropriate use of personal protective equipment (PPE) in hospitals is in constant flux as research into SARS COV-2 transmission continues to develop our understanding of the virus. The risk associated with procedures classed as 'aerosol generating' is a subject that is in constant debate. Current guidance is largely based on pragmatic and cautious logic, as the scientific evidence of aerosolization and transmission of respiratory viruses associated with procedures is scarce. The physical properties of aerosol particles which may contain viable virus, have implications for the safe use of PPE and infection control protocols. As elective work in the NHS is reinstated it is important that the implications associated with the possibility of airborne transmission of the virus in hospitals is more widely understood. This will facilitate appropriate use of PPE and help direct further research into the true risks of aerosolization during these procedures to allow safe streamlining of services for staff and patients.

Title:

An anaesthetic and intensive care perspective on infection control measures for the prevention of airborne transmission of SARS CoV-2

Key words: SARS CoV-2, Covid19, aerosol generating procedures, Personal Protective equipment, aerosol,

\section{Abstract}


Guidance regarding appropriate use of personal protective equipment (PPE) in hospitals is in constant flux as research into SARS COV-2 transmission continues to develop our understanding of the virus. The risk associated with procedures classed as 'aerosol generating' is a subject that is in constant debate. Current guidance is largely based on pragmatic and cautious logic, as the scientific evidence of aerosolization and transmission of respiratory viruses associated with procedures is scarce. The physical properties of aerosol particles which may contain viable virus, have implications for the safe use of PPE and infection control protocols. As elective work in the NHS is reinstated it is important that the implications associated with the possibility of airborne transmission of the virus in hospitals is more widely understood. This will facilitate appropriate use of PPE and help direct further research into the true risks of aerosolization during these procedures to allow safe streamlining of services for staff and patients.

\section{Key Points}

- Aerosols are particles that are smaller than droplets, and remain airborne for longer periods of time.

- If aerosols contain viable virus, prolonged suspension will mean that the environment in which they were generated carries a risk of airborne transmission for an unknown length of time, and allows for deposition of the virus over a larger radius. 
- The definition of an aerosol generating procedure (AGP) is a procedure that generates aerosols over and above those generated during normal speech, coughing and sneezing, and is associated with increased risk of pathogen transmission to healthcare workers.

- The evidence pertaining to the risk of aerosol generation, and transmission of viruses due to procedures that are classed as aerosol generating is scarce, therefore guidance is largely based on pragmatic and cautious logic.

- Use of PPE and infection control measures, especially for prevention of airborne transmission, can create delays and barriers to care.

- More research into risks of procedures currently classified as AGPs, especially in asymptomatic patients may help streamline future services. 


\section{Introduction}

The prevalence of SARS CoV-2 continues to necessitate daily consideration of heightened infection control precautions for all healthcare workers (HCWs). Personal protective equipment (PPE) has been written about extensively in the media and medical journals. In particular, the concept of aerosol generating procedures (AGPs) and appropriate measures required to minimise associated transmission, is point of regular debate.

As elective work is reinstated, risk management and appropriate PPE strategies require careful scrutiny. In addition, extrapolation from epidemiological modelling regarding a second spike in infection rates(Xu and Li 2020) necessitates readying the workforce for another period of redeployment and high volume contact with confirmed or suspected SARS-CoV-2 infection. It is important to have clear, effective infection control and PPE strategies. However, as understanding of the virus develops, the guidance regarding infection control practice is in a constant state of flux. For this reason, it is also imperative that the rationale behind practices is understood.

\section{Aerosol Generating Procedures (AGP) and airborne transmission}

Transmission of SARS CoV-2 occurs mainly via the droplet or contact route. Generation of aerosols, creates the possibility of airborne transmission. The WHO defines an AGP as "any medical and patient care procedure that results in the production of airborne particles (aerosols)"'(WHO, 2014). However, breathing, speaking and coughing can generate aerosols from the respiratory tract( Yang et al 2007; Anfinrud et al 2020). Aerosolised particulates 
containing SARS CoV-2 RNA have been detected in hospital areas where no AGPs take place(Liu et al 2020) leading to speculation that airborne transmission may be possible outside hospital settings. The risk of airborne transmission may be increased significantly during the performance of 'high risk AGPs', namely "medical procedures that have been reported to be aerosol-generating and consistently associated with an increased risk of pathogen transmission"(World Health Organisation, 2014). A more helpful definition of an AGP might be; a procedure which are 'more likely to generate higher concentrations of infectious respiratory aerosols than coughing, sneezing, talking, or breathing' and 'potentially put healthcare personnel and others at an increased risk for pathogen exposure and infection' offered by the Centers for Disease Control and Prevention (CDC 2020) in the United States.

Aerosols are generated by movement of air across the surface of a liquid. Increased force of air currents decreases the size of particles generated.(WHO, 2014) Procedures which result in high velocity airflow at the interface between air and the respiratory mucosa of infected patients, beyond that of a cough or sneeze, likely fulfil these criteria. It is of particular relevance to SARS CoV-2 transmission, as infected individuals accumulate high viral loads in upper airway secretions.(To et al 2020)

The risk associated with AGPs rests on the behaviour of aerosolised particulates. An aerosol is a particle suspended in a gas that is small enough to remain airborne for long periods of time. By convention a cut off of $5 \mu \mathrm{m}$ is often used, with particles of this diameter or smaller classed as aerosols(Tellier 2006). In contrast, droplets are particles of a critical size at or 
above which they settle on surfaces quickly. Particles of $10 \mu \mathrm{m}$ diameter or more generally fall into this category. Between 5 and $10 \mu \mathrm{m}$ particles behave as either aerosols or droplets depending on environmental factors(Kohanski et al 2020). The presence of infective aerosols increases the radius of air and surfaces around patients that should be considered contaminated. In an indoor environment, a $10 \mu \mathrm{m}$ diameter particle will settle $1 \mathrm{~m}$ in 5.6 minutes, whereas a particle of $1 \mu \mathrm{m}$ diameter settles $1 \mathrm{~m}$ in 9.3 hours(Kohanski et al 2020). The longer a particle remains airborne, the further it may travel from its origin. Particles behaving as droplets tend to settle within 1.5 to $2 \mathrm{~m}$ of their origin, hence droplet precautions are required within $2 \mathrm{~m}$ of patients.(Tran et al 2012) The length of time prior to settling determines how long air remains contaminated, though this will be impacted by local ventilation and air currents. Particle size also affects deposition site within the respiratory tract. Particles of $5 \mu \mathrm{m}$ or below generally deposit in the lower airways. Droplets larger than $10 \mu \mathrm{m}$ are more likely to deposit in the upper airways.(Tellier 2006) The impact of anatomical point of deposition is not known. Theoretically, deposition lower in the respiratory tract could affect the type and severity of symptoms experienced.

\section{Which procedures are aerosol generating?}

Table 1 lists the procedures currently classed by Public Health England (PHE) as 'aerosol generating procedures'. The absence of chest compressions during cardiopulmonary resuscitation (CPR) from this list diverges from guidance issued by the Resuscitation Council UK, which classes it as an AGP (J Wyllie RCUK President, 2020). Data surrounding transmission risks associated with CPR are rife with confounding factors. It is not possible to separate the risks of different procedures performed during CPR, including tracheal 
intubation and manual ventilation. As such, guidance must be based on pragmatism and logic. The UK's New and Emerging Respiratory Virus Threats Advisory Group (NERVTAG) argues that during chest compressions, airflow through the patient's respiratory tract will be akin to that of breathing, therefore should not be treated as an AGP.(PHE, 2020a) In fact, all procedures in the table 1 have varying, generally weak levels of evidence supporting their designation as aerosol generating. As such, advisory bodies must take a pragmatic and cautious approach.

\section{Intubation/extubation}

One study investigating particle sizes containing H1N1 RNA before and after intubation of infected patients, found increased particles of diameters that would be considered aerosols after intubation, though this was not statistically significant(Thompson et al 2013). Several small studies investigating transmission of SARS and other respiratory viruses to HCWs found a weak association with performance of intubation and infection. All point to confounding factors, including difficulty in confirming use of droplet PPE and contact precautions(NSS, 2020). During the SARS epidemic, a small study found HCWs involved in intubation showed higher rates of infection compared to controls.(Fowler et al 2004) Nurses involved in patient care during the peri-intubation period were at highest risk, raising the possibility that the risk to HCWs is increased by exposure to the critically ill patient and surroundings prior to intubation. This raises questions pertaining to the risk of intubation in asymptomatic, swab negative patients, requiring further investigation. Recent data suggests a low rate of infection with SARS CoV-2 in intensivists and anaesthetists undertaking 
endotracheal intubation(Cook 2020). It is not possible to conclude whether this reflects adequate PPE use during intubation, or that the risk during intubation has been overstated.

Logically it is difficult to ascertain a mechanism of aerosol formation during tracheal intubation. There is little to no airflow in the respiratory tract of patients who have received neuromuscular blocking agents during the act of tube insertion. There may be increased risk associated with leaks during face mask ventilation. It is very difficult and not of particular use to separate the risk associated with these procedures, as it is rare to perform one without the other, especially in the critically ill. Interestingly, little to no literature exists about risk during extubation, which logically presents more opportunity for aerosolization. Extubation, like deep respiratory suction, can instigate forceful coughing. The removal of the endotracheal tube, if high flows continue through the circuit, will allow aerosolization of secretions deposited on the tube.

\section{Tracheostomy}

Insertion of tracheostomy during the SARS epidemic showed increased transmission risk to HCWs involved compared to controls(Tran et al 2012). No evidence specifically pertaining to the generation of aerosols during this procedure exists, however, the process will often involve bronchoscopy guidance creating a profound leak from the ventilation circuit distal to microbial filters. This would most likely be the source of aerosol generation. Disconnection while moving from endotracheal tube to tracheostomy also provides opportunity for aerosol generation. Tracheostomy change or removal in unparalysed patients usually involves suctioning and will cause forceful coughing which may generate aerosolised particles from upper airways and the tracheostomy site. 


\section{Bronchoscopy}

There are few weak and contradictive air sampling studies investigating aerosolization during bronchoscopy (NSS, 2020). One study concluded bronchoscopy without delivery of nebulised medication did not generate more aerosols(O'Neil et al, 2017). However, a study of bronchoscopy focusing on influenza RNA, detected increased RNA containing aerosols, though this was underpowered(Thompson et al, 2013). In the absence of conclusive evidence, treating bronchoscopy as an AGP remains a sensible precaution.

\section{Non-invasive ventilation}

Both CPAP (continuous positive airway pressure) and BiPAP (Bilevel Positive airway pressure) use high flow gas to apply positive pressure via a tight-fitting mask or hood. Air inevitably leaks around the mask, especially when ill fitting, providing opportunity for aerosol dispersion. Evidence pertaining to the use of BiPAP during the SARS epidemic shows a weak association with infection in HCW (NSS, 2020), however, one particle sampling study concluded that there was no increase in aerosolisation when using BiPAP(Simonds et al, 2010). Other studies investigating BiPAP delivery concur that it increases the radius of exhaled air dispersion, with higher inspiratory pressures further increasing the dispersion radius to up to $1 \mathrm{~m}$ ( Hui et al 2006; Hui et al 2014) These studies did not investigate particle size or number thus can only conclude that the aerosols generated by normal exhalation are spread over a wider area, not that there is increased aerosol generation. No studies specific to the delivery of CPAP were found on literature search. 


\section{High Flow Nasal Oxygen (HFNO)}

Delivery of flows of up to 60 litres per minute can be achieved using HFNO devices. Mouth opening allows flow from this to be diverted out of the oral cavity, creating a mechanism for aerosol generation from the nasal passages and upper airways.(Kotoda et al 2020) Evidence of aerosol generation by HFNO is mainly based on in vitro studies(Kotoda et al 2020; Roberts 2015). One in vivo study using patients with bacterial pneumonia, concluded that HFNO did not create greater airborne contamination in comparison to conventional oxygen therapy.(Leung et al 2019) However, bacterial and viral contamination are not directly comparable, and this was performed in an environment with 6 air changes per hour rendering the results difficult to apply to ward environments.

A recent review concluded that evidence pertaining to smoke particle dispersion at HFNO of 60 litres per minute was comparable to that of the use of oxygen masks at 15 litres per minute.(Li et al 2020) This investigated only particles $<1 \mu \mathrm{m}$ in diameter so again is not conclusive evidence of safety. It was, however, noted that the patient donning a surgical face mask in conjunction with HFNO can significantly reduce dispersion of bioaerosols and is worth considering as a risk reduction strategy.

\section{High speed cutting devices (post-mortem, surgery, dental)}

Multiple studies have concluded that high-speed cutting devices used in surgery and postmortems generate aerosols. Dental procedures using ultrasonic and sonic drilling also generate aerosols (NSS, 2020). If the aerosol source is the respiratory tract or upper airways, airborne PPE precautions should be worn. There are concerns regarding the possibility of 
infectivity of smoke plumes and gas released after laparoscopy(RCSEng. 2020a) but there is currently no evidence to support this.

\section{Airway suctioning}

The evidence base for risk associated with airways suctioning involves HCW infection rates during the SARS epidemic (NSS, 2020). These data pertain to the suctioning of intubated patients, and involve ventilator circuit disconnection, which is the logical source of aerosolization.

\section{Induction of sputum and nebulisation}

Multiple studies have concluded that chest physiotherapy is neither associated with an increased transmission of SARS nor increased aerosol particle detection(NSS, 2020). There is evidence that although nebulisation increases aerosol particle detection, these particles are generated from the nebuliser, not the patient, therefore would not pose an infection risk. Despite this, induction of sputum with nebulised saline remains classified as an AGP. Forceful and prolonged coughing will generate aerosols, although increased risk of transmission has not been demonstrated. Governing bodies are issuing precautionary guidance surrounding procedures that involve proximity to the airway of patients undergoing procedures that induce this. The Royal College of Surgeons has stated that nasogastric tube insertion, which often causes forceful coughing, should be considered an AGP (HPS, 2020). Furthermore, the Royal College of Speech and Language therapists asserts 
that procedures such as swallow assessment inducing forceful coughing, pose similar infection risks to induction of sputum using nebulised saline. (Bolton L et al, 2020)

\section{Personal protective equipment}

Current PPE guidance falls into two categories, droplet precautions, required within $2 \mathrm{~m}$ of patients, and airborne precautions required where AGPs are performed. Droplet precautions include sessional use of fluid resistant surgical masks and eye protection, single use gloves and plastic aprons. Patients should also wear a fluid resistant surgical mask, as the mechanism of risk reduction is mainly via reduction of droplet dispersal from the mask wearer (Rodriguez-Palacios et al 2020).

Recommended PPE for environments with risk of aerosolisation include single use of filtering face piece respirator 3 (FFP3) masks, which should filter aerosolised particles at $>99 \%$ efficiency, eye protection, long-sleeved fluid resistant gown, and gloves. Long-sleeved gowns are required as bioaerosols may contaminate larger surface areas of clothing. Transmission occurs when an HCW touches contaminated clothing then touches their mouth nose or eyes. The gown reduces this risk by removing contamination once contact with the high-risk environment is terminated. PHE guidance makes no mention of hair coverings, they are unlikely to afford extra protection in aerosolised environments as the microbiome on human hair should rapidly destroy viral particles settling on hair. (IPS, 2020) One important consideration regarding PPE use is identifying areas that are high risk and for how long these areas should be treated as containing aerosols, therefore requiring airborne 
PPE precautions, after AGP performance. Current guidance regarding timing of aerosol clearance after AGP is based on the number of air changes in the room. 5 air changes are sufficient to remove $>99 \%$ of airborne contaminants. (RCSEng, 2020b) This guidance is simple to follow in controlled environments such as operating theatres or negative pressure rooms, where air exchange rates are controlled. Most operating theatres undergo 5 air change within 15 minutes after an AGP, rendering the room safe for HCWs to enter in droplet precaution PPE. However, little attention in the literature has been paid to remote areas where AGPs are performed less frequently. During surges, some areas such as resuscitation bays in the emergency department, can be cohorted with HCWs consistently wearing airborne precautions in these areas. As admissions for SARS CoV-2 infections decrease it is not viable to continue this practice. This creates issues when an AGP must be performed in an area such as this, or a ward area, where ventilation rates are unknown. How long these areas are contaminated with aerosols and the radius and timing of surface decontamination optimal for risk reduction, must be elucidated.

Bearing this in mind, in ICU HCWs are required to wear airborne precaution PPE for all contact with potentially infected ventilated patients. This presumably is to ensure that in the event of accidental ventilator disconnection distal to the microbial filter, HCWs are still protected. It is of note that similar precautions are not required in operating theatres where patients are also on closed circuit ventilation.

Problems posed by personal protective equipment and infection prevention and control strategies for anaesthesia and intensive care 
As specialties used to dealing with emergencies, intensivists and anaesthetists understand the importance of communication. One of the biggest challenges during the pandemic has been difficulties faced by HCWs in communicating while wearing PPE for AGPs. The deciphering of facial expressions and lip reading is rendered impossible while speech is muffled by tight-fitting masks and visors. The extra bandwidth occupied by fear of breaching PPE guidance, accompanied by discomfort and increased heat, makes normal working more physically and mentally demanding. This becomes compounded in emergency situations. Any safe reduction in PPE requirements could therefore be of benefit to patients and staff.

\section{Theatre turnover}

As elective surgical work is reintroduced, waiting lists are longer than ever, and increasing list turnover in theatres will be paramount in tackling the backlog. This may be hampered by IPC measures including the donning and doffing of PPE and the need for delays after each AGP, before HCWs can enter to commence surgery or decontaminate the area. This delay can be mitigated by staff wearing airborne precaution PPE for the entire theatre session, but the aforementioned disadvantages of PPE in team environments must be weighed against the time savings that could be made.

Consensus guidance from Royal Colleges and PHE on August $20^{\text {th }} 2020$ downgraded operating theatres from a 'high risk hotspot'. Airborne precaution PPE and delays for air changes are not required for AGPs in asymptomatic patients with a negative swab within 72 hours of surgery(PHE, 2020b). However, it is not always possible to fulfil these guidelines, and if background rates of infection increase, precautions may be reinstated. Guidance 
suggests responsibility for this decision be left to individual hospitals(RCSEng, 2020b). It will be difficult for hospitals to make defensible decisions without increased mass testing to provide robust data about local SARS CoV-2 prevalence.

\section{Intensive care}

As ICUs cease providing care for SARS CoV-2 positive patients in cohorted areas, issues associated with isolation rooms requiring airborne precaution PPE become highlighted. These patients may be reviewed in person and examined less often, both by ICU physicians and other specialties, in attempt to reduce the use of PPE and minimise staff exposure. Meticulous communication between staff inside and outside of the room is required, and careful evaluation of the need for patient examination must be weighed against the risks of contamination and excessive PPE use.

\section{Conclusion and further research}

This analysis of the risks surrounding aerosol generation in hospitals highlights the paucity of evidence on which experts can base guidance. As services that were paused during the height of the first wave of SARS CoV-2 infection are reinstated, the safety of HCWs and patients is paramount. These considerations are sometimes at odds with each other, as PPE use creates issues regarding patient care and dealing with important backlogs in surgical work, which if left unchecked could risk lives. It is important that while patient pathways are redesigned, robust data is collected investigating the risks surrounding procedures believed to be aerosol generating. 
Tracking infection rates among healthcare professionals, and correlating this with PPE use, will identify inadequate PPE use and guidelines that are insufficient for HCW protection. If current guidance is adhered to correctly, however, these outcomes will be of no use in identifying overuse of PPE or if unnecessary delays in theatres are occurring. For the downgrading of PPE use in elective settings, two important areas of research are required. Robust evidence of ongoing SARS CoV-2 prevalence in local populations must be gathered using mass testing. In addition, air sampling studies in theatres, to understand the true risk of aerosolization during performance of procedures classed as aerosol generating, especially in asymptomatic patients, may help elucidate appropriate infection prevention strategies. 
Tables

Table 1: list of procedures classed as aerosol generating as per Public Health England guidance August 2020 (PHE 2020b).

\begin{tabular}{|l|}
\hline Aerosol Generating Procedures \\
\hline Respiratory tract suctioning \\
\hline Bronchoscopy \\
\hline Manual ventilation \\
\hline Tracheal intubation and extubation \\
\hline Tracheotomy or tracheostomy procedures (insertion or removal) \\
\hline Upper ENT airway procedures that involve suctioning \\
\hline Upper gastro-intestinal endoscopy where there is open suctioning of the upper respiratory \\
tract \\
\hline High speed cutting in surgery/post-mortem procedures if this involves the respiratory tract \\
or paranasal sinuses \\
\hline Dental procedures using high speed devices such as ultrasonic scalers and high-speed drills \\
\hline Non-invasive ventilation (NIV); Bi-level Positive Airway Pressure Ventilation (BiPAP) and \\
Continuous Positive Airway Pressure Ventilation (CPAP) \\
\hline High Frequency Oscillatory Ventilation (HFOV) \\
\hline Induction of sputum using nebulised saline \\
\hline High flow nasal oxygen (HFNO) \\
\hline
\end{tabular}




\section{References}

WHO (World Health Organisation). 2014. Infection Prevention and Control of Epidemic- and Pandemic-Prone Acute Respiratory Infections in HealthCare. https://apps.who.int/iris/bitstream/handle/10665/112656/9789241507134_eng.pdf ;jsessionid=3215A9ADF83E693E43D51BC7B4FC18F4?sequence=1 (accessed $25 / 8 / 2020)$

Anfinrud P, Stadnytskyi V, Bax CE, Bax A. 2020. Visualizing Speech-Generated Oral Fluid Droplets with Laser Light Scattering. N Engl J Med 382(21):2061-2063.

Bolton L, Mills C, Wallace S, Brady M, Royal College of Speech and Language Therapists COVID-19 Advisory Group. Aerosol generating prcedures 2020, dysphagia assessment and COVID-19 https://www.rcslt.org/-/media/docs/Covid/RCSLT-Dysphagia-andAGP220420FINAL-1-(1).PDF?la=en\&hash=816B77BE5A88976CD97F32B84754F223FA761C54 (accessed 25/8/2020)

CDC (centres for disease control) 2020 August. Clinical Questions about COVID-19: Questions and Answers https://www.cdc.gov/coronavirus/2019-ncov/hcp/faq.html (accessed 3/9/2020) 
Cook T. M. (2020). Risk to health from COVID-19 for anaesthetists and intensivists - a narrative review. Anaesthesia, 10.1111/anae.15220. Advance online publication. https://doi.org/10.1111/anae.15220

Fowler RA, Guest CB, Lapinsky SE, Sibbald WJ, Louie M, Tang P, Simor AE, Stewart TE. 2004. Transmission of severe acute respiratory syndrome during intubation and mechanical ventilation. Am J Respir Crit Care Med 169(11):1198-202.

Hui DS, Chan MT, Chow B. 2014. Aerosol dispersion during various respiratory therapies: a risk assessment model of nosocomial infection to health care workers. Hong Kong Med J 20 Suppl 4:9-13.

Hui DS, Hall SD, Chan MT, Chow BK, Tsou JY, Joynt GM, Sullivan CE, Sung JJ. 2006. Noninvasive positive-pressure ventilation: An experimental model to assess air and particle dispersion. Chest 130(3):730-40.

HPS (Health Protection Scotland). 2020. Review of national and international guidance on infection prevention and control measures for Personal Protective Equipment (PPE) and Aerosol Generating Proceedures (AGPs) for COVID-19. https://hpspubsrepo.blob.core.windows.net/hps-website/nss/3048/documents/1 covid-19ipc-guidance-comparison-for-ppe.pdf (accessed 26/8/2020) 
IPS (Infection Prevention Society). 2020. Using Personal Protective Equipment for Covid-19 in Intensive Care Settings: Questions and Answers https://cdn.ps.emap.com/wpcontent/uploads/sites/3/2020/05/IPS-Use-of-PPE-in-ICU.pdf (accessed 25/8/2020)

Kohanski MA, Lo LJ, Waring MS. 2020. Review of indoor aerosol generation, transport, and control in the context of COVID-19. Int Forum Allergy Rhinol.

Kotoda M, Hishiyama S, Mitsui K, Tanikawa T, Morikawa S, Takamino A, Matsukawa T. 2020. Assessment of the potential for pathogen dispersal during high-flow nasal therapy. J Hosp Infect 104(4):534-537.

Leung CCH, Joynt GM, Gomersall CD, Wong WT, Lee A, Ling L, Chan PKS, Lui PCW, Tsoi PCY, Ling CM et al. 2019. Comparison of high-flow nasal cannula versus oxygen face mask for environmental bacterial contamination in critically ill pneumonia patients: a randomized controlled crossover trial. J Hosp Infect 101(1):84-87.

Li J, Fink JB, Ehrmann S. 2020. High-flow nasal cannula for COVID-19 patients: low risk of bioaerosol dispersion. Eur Respir J 55(5).

Liu Y, Ning Z, Chen Y, Guo M, Gali NK, Sun L, Duan Y, Cai J, Westerdahl D, Liu X et al. 2020. Aerodynamic analysis of SARS-CoV-2 in two Wuhan hospitals. Nature 582(7813):557-560.

NSS (National Services Scotland). 2020. Supplimentary document 1, assessing the evidence base for medical proceedures which may create a higher risk of respiratory infection 
transmission from patient to healthcare worker.

https://hpspubsrepo.blob.core.windows.net/hps-website/nss/3055/documents/2 agpsupplmentary-document.pdf (accessed 3 Sept 2020)

O'Neil CA, Li J, Leavey A, Wang Y, Hink M, Wallace M, Biswas P, Burnham CD, Babcock HM, Program CfDCaPE. 2017. Characterization of Aerosols Generated During Patient Care Activities. Clin Infect Dis 65(8):1335-1341.

PHE (Public Health England). 2020a. PHE statement regarding NERVTAG review and consensus on cardiopulmonary resuscitation as an aerosol generating procedure (AGP) https://www.gov.uk/government/publications/wuhan-novel-coronavirus-infectionprevention-and-control/phe-statement-regarding-nervtag-review-and-consensus-oncardiopulmonary-resuscitation-as-an-aerosol-generating-procedure-agp--2 laccessed $\underline{24 / 8 / 2020)}$

PHE (Public Health England). 2020b. COVID-19: Guidance for the remobilisation of services within health and care settings.

https://assets.publishing.service.gov.uk/government/uploads/system/uploads/attachment data/file/910885/COVID-

19 Infection prevention and control guidance FINAL PDF 20082020.pdf (accessed $25 / 8 / 2020)$

Roberts SaKNaSCaODJaAZaDRaRSaJYaJM. 2015. Nasal high-flow therapy and dispersion of nasal aerosols in an experimental setting. Journal of Critical Care 30:842. 
Rodriguez-Palacios A, Cominelli F, Basson AR, Pizarro TT, llic S. 2020. Textile Masks and Surface Covers-A Spray Simulation Method and a "Universal Droplet Reduction Model" Against Respiratory Pandemics. Front Med (Lausanne) 7:260.

RCSEng (Royal College of Surgeons of England). 2020a. Updated Intercollegiate General Surgery Guidance on COVID-19 https://www.rcseng.ac.uk/coronavirus/joint-guidance-forsurgeons-v2/ (accessed 26/8/2020)

RCSEng (Royal College of Surgeons of England). 2020b. Managing theatre processes for planned surgery between COVID-19 surges, https://icmanaesthesiacovid-19.org/managingtheatre-processes-for-planned-surgery-between-covid-19-surges (accessed 25/8/2020)

Simonds AK, Hanak A, Chatwin M, Morrell M, Hall A, Parker KH, Siggers JH, Dickinson RJ. 2010. Evaluation of droplet dispersion during non-invasive ventilation, oxygen therapy, nebuliser treatment and chest physiotherapy in clinical practice: implications for management of pandemic influenza and other airborne infections. Health Technol Assess 14(46):131-172.

Tellier R. 2006. Review of aerosol transmission of influenza A virus. Emerg Infect Dis 12(11):1657-62. 
Thompson KA, Pappachan JV, Bennett AM, Mittal H, Macken S, Dove BK, Nguyen-Van-Tam JS, Copley VR, O'Brien S, Hoffman P et al. 2013. Influenza aerosols in UK hospitals during the H1N1 (2009) pandemic--the risk of aerosol generation during medical procedures. PLoS One 8(2):e56278.

To KK, Tsang OT, Leung WS, Tam AR, Wu TC, Lung DC, Yip CC, Cai JP, Chan JM, Chik TS et al. . 2020. Temporal profiles of viral load in posterior oropharyngeal saliva samples and serum antibody responses during infection by SARS-CoV-2: an observational cohort study. Lancet Infect Dis 20(5):565-574.

Tran K, Cimon K, Severn M, Pessoa-Silva CL, Conly J. 2012. Aerosol generating procedures and risk of transmission of acute respiratory infections to healthcare workers: a systematic review. PLoS One 7(4):e35797.

Wyllie J, RCUK President. 2020 April. RCUK Statement on PHE PPE Guidance https://www.resus.org.uk/about-us/news-and-events/rcuk-statement-phe-ppe-guidance (accessed 3 Sept 2020)

Xu S, Li Y. 2020. Beware of the second wave of COVID-19. Lancet 395(10233):1321-1322.

Yang S, Lee GW, Chen CM, Wu CC, Yu KP. 2007. The size and concentration of droplets generated by coughing in human subjects. J Aerosol Med 20(4):484-94. 